\title{
Tecnura
}

\section{Análisis dee velocidad de transmisión del protocolo AX.25 Implementado en la estación terrena satelital UPTC}

\section{Analysis of transmission speed of AX.25 Protocol implemented in satellital earth station UPTC}

\author{
Oscar Fernando Vera Cely, Jairo Alonso Mesa Lara, Roberto Ferro Escobar
}

Fecha de recepción: 12 de abril de 2015

Fecha de aceptación: 24 de agosto de 2015

Como citar: Vera Cely, O. F., Mesa Lara, J. A., \& Ferro Escobar, R. (2015). Analisis de velocidad de transmisión del protocolo AX.25 implementado en la estación terrena satelital UPTC. Revista Tecnura, 19(46), 93-102. doi:10.14483/udistrital.jour.tecnura.2015.4.a07

\section{Resumen}

Uno de los parámetros importantes para el correcto funcionamiento de la estación terrena satelital proyectada para la Universidad Pedagógica y Tecnológica de Colombia (UPTC) es la eficiencia en la velocidad de transmisión con el protocolo de comunicaciones. Este artículo presenta los resultados del análisis de la velocidad de transmisión del protocolo AX.25 que se encuentra implementado en el sistema de comunicación de la estación terrena satelital UPTC. Inicialmente se hace una breve descripción del hardware adquirido para la estación; se evalúa el comportamiento de la velocidad de transmisión por medio de un análisis teórico basado en ecuaciones que permiten estimar este parámetro en el funcionamiento del protocolo, posteriormente se realizan pruebas utilizando los equipos con que cuenta la estación terrena UPTC y por último, se presentan las conclusiones. A partir de la comparación del análisis teórico con resultados obtenidos de manera experimental, se evidenció que la eficiencia del protocolo AX.25 es mayor en la medida que aumenta el número de tramas.
Palabras clave: Estación terrena satelital, protocolo AX.25, velocidad de transmisión.

\begin{abstract}
One of the important parameters for the proper functioning of satellital ground station projected on Pedagogical and Technological University of Colombia (UPTC) is the efficiency in transmission speed on communications protocol. This paper shows the results of analysis of the transmission speed of the AX.25 protocol implemented in the communication system of the satellital ground station UPTC. It begins with a brief description of the implemented hardware; the behavior of the transmission rate is evaluated using a theoretical analysis based on equations to estimate this parameter in the operation of the proto$\mathrm{col}$, then tests are performed using the hardware that the satellital ground station UPTC has and finally, the conclusions are presented. Based on comparison of the theoretical analysis results obtained experimentaIly, it became apparent that AX.25 protocol efficiency is higher when increasing the number of frames.
\end{abstract}

Keywords: AX.25 protocol, transmission speed, sateIlital earth station

\footnotetext{
Ingeniero Electrónico, magíster en Tecnología Informática. Docente de la Universidad Pedagógica y Tecnológica de Colombia. Tunja, Colombia. Contacto: oscar.vera@uptc.edu.co

2 Ingeniero Electrónico, especialista en Diseño y Construcción de Soluciones Telemáticas, magíster en Ciencias de la Información y las Comunicaciones. Docente de la Universidad Pedagógica y Tecnológica de Colombia. Contacto: jairo.mesa@uptc.edu.co

3 Ingeniero Electrónico, magíster en Teleinformática, doctor en Ingeniería Informática. Docente de la Universidad Distrital Francisco José de Caldas. Bogotá, Colombia. Contacto: ferro.roberto@gmail.com
} 


\section{INTRODUCCIÓN}

La UPTC cuenta con una estación terrena satelital, la cual fue diseñada siguiendo el esquema tradicional monolítico (Espindola, Nonsoque, \& Molano, 2012), conformado por los siguientes elementos: antenas, mecanismo de servomotor, transceptor y módem. La unidad de control del sistema de comunicación se encuentra en el transceptor kenwood TM-D700, que a su vez realiza las funciones de módem; este dispositivo cuenta con un controlador de nodo terminal (TNC), el cual proporciona la implementación del protocolo AX.25 para llevar a cabo los enlaces de comunicación.

Un aspecto importante en un sistema de comunicación es el protocolo con el que se transmitirá la información, ya que este es el que permitirá hacer una codificación de los datos con el fin de tener bajas pérdidas de información. El protocolo AX.25, que apareció como una versión del protocolo X.25 (Parry, 1997), se ha convertido en el más usado en el área de comunicaciones satelitales para radioaficionados (Diersing \& Ward, 1989). La banda del espectro para radioaficionado permite realizar pruebas de carácter académico, razón por la cual se trabaja sobre esta banda para el desarrollo de investigaciones en el área. En la región de Boyacá no se han realizado análisis de este tipo, es por esto que este trabajo aporta avances en el proyecto del montaje y puesta en marcha de la estación terrena UPTC.

Se propone el análisis de velocidad del protocolo AX.25 en el sistema de comunicaciones de la estación terrena satelital de la UPTC. Dicho estudio se realizó con base en los conceptos plasmados en la sección 2, utiliza los métodos y materiales descritos en la sección 3. En la sección 4 se presentan los resultados obtenidos a partir de las pruebas realizadas, mientras que la discusión y el aporte de este trabajo a la investigación en comunicaciones satelitales en la región de Boyacá se muestra en la sección 5 . Finalmente se presenta la conclusión en la sección 6.

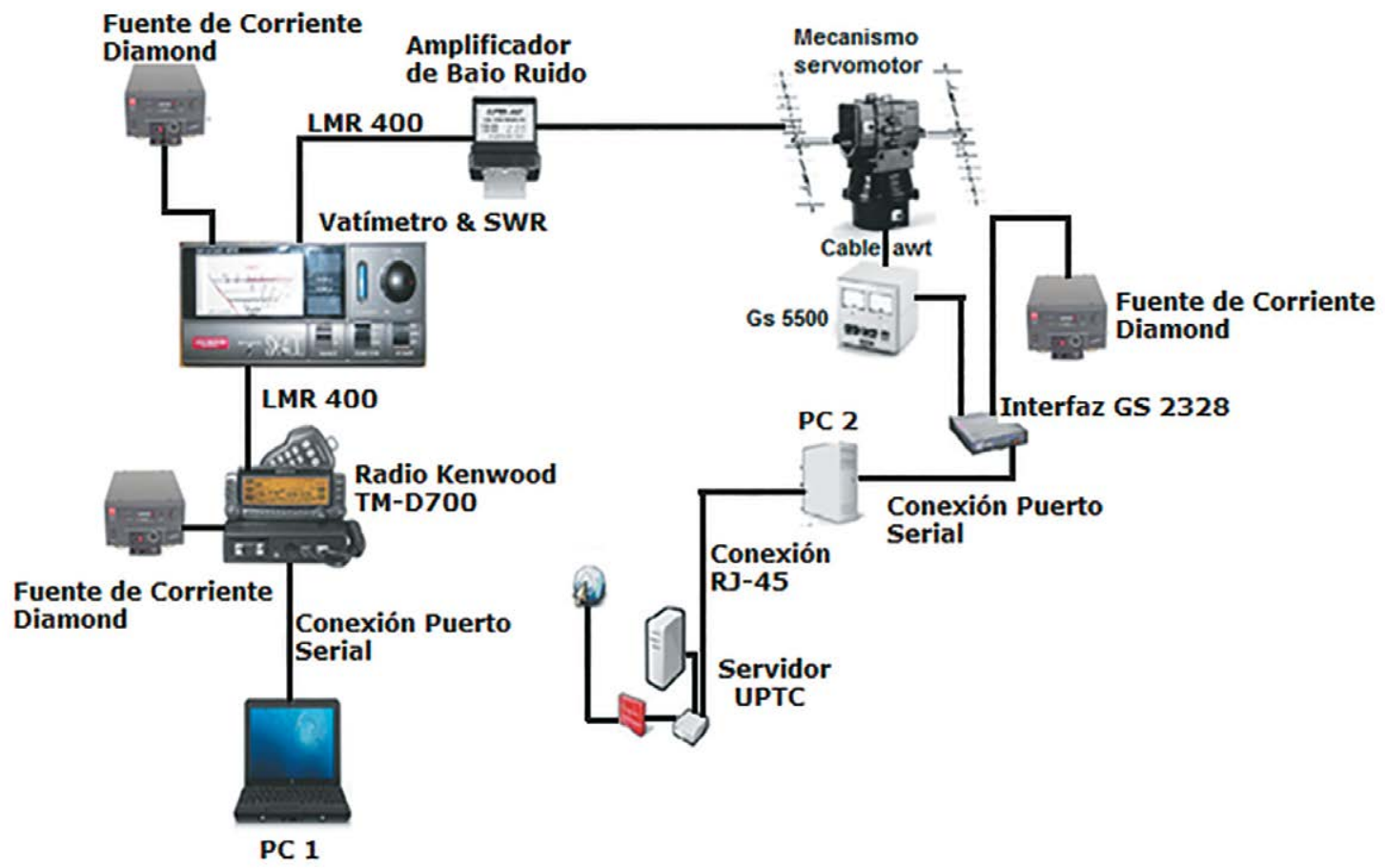

Figura 1. Esquema general del hardware de la estación terrena satelital UPTC.

Fuente: Espíndola, Nonsoque, y Molano (2012) 


\section{MARCO TEÓRICO}

La figura 1 muestra el esquema general del hardware que se encuentra implementado en la estación terrena satelital.

Para el desarrollo de este trabajo se hizo énfasis en la parte correspondiente al transceptor Kenwood TM-D700, que es el dispositivo directamente relacionado con el protocolo AX.25.

Los sistemas de comunicaciones para la comunidad de radioaficionados utilizan el protocolo AX.25, que se creó por la necesidad de esta comunidad de poder recibir y transmitir datos sobre varios enlaces de comunicaciones entre dos terminales. El AX.25 versión 2.2 es un protocolo ubicado en la capa de enlace que suministra dicho servicio, independiente de la existencia de capas superiores en el sistema de comunicación (Beech, Nielsen, \& Taylor, 1997).

\section{Modelo del protocolo AX.25}

El modelo del protocolo AX.25 está basado en el modelo de referencia OSI, el cual se conforma de capas, donde cada una provee un punto de acceso de servicio (SAP) para la interfaz de la capa superior (figura 2).

\begin{tabular}{c|c|} 
Layer & Function \\
\cline { 2 - 2 } & Application \\
\hline 6 & Presentation \\
\hline 5 & Session \\
\hline 4 & Transport \\
\hline 3 & Network \\
\hline 2 & Data Link \\
\hline 1 & Physical \\
\hline
\end{tabular}

Figura 2. Modelo de referencia OSI

Fuente: Beech, Nielsen, \& Taylor (1997).
A partir de las capas de enlace de datos (capa 2) y de la capa física (capa 1) se modela el protocolo AX.25, en el cual estas dos capas se subdividen en varias máquinas de estado finito (figura 3 ).

En la figura 3, la función Data-Link Service Access Point (DLSAP) indica un punto de acceso a servicio de enlace de datos, esta función proporciona el servicio que combina las funciones de la capa física y la capa de enlace de datos. La función principal del DLSAP se centra en el enlace de datos (Data Link), esta máquina de estado suministra la lógica necesaria para establecer conexiones entre dos estaciones e intercambiar información (Beech, Nielsen, \& Taylor, 1997).

Dentro del DLSAP, el segmentado se encarga de aceptar información proveniente de las capas superiores; en caso de que los datos excedan el límite de tamaño de las tramas soportadas por el protocolo AX.25, serán divididas en segmentos más pequeños de tal forma que puedan ser reconstruidos luego de completar el proceso en el enlace AX.25 (Beech, Nielsen, \& Taylor, 1997).

La máquina de estado correspondiente a la gestión del enlace de datos (Management Data Link) se encarga de proporcionar los parámetros de negociación del protocolo AX.25, y gestiona la lógica necesaria para establecer la negociación entre dos estaciones (Beech, Nielsen, \& Taylor, 1997).

La función que cumple la máquina de estado de multiplexado (Link Multiplexer) permite tener varios enlaces al mismo tiempo sobre el mismo canal

\begin{tabular}{|c|c|}
\hline Layer & Function(s) \\
\hline \multirow{3}{*}{ Data Link (2) } & Management \\
\hline & Data Link \\
\hline & Link Multiplexer \\
\hline \multirow{2}{*}{ Physical (1) } & Physical \\
\hline & Silicon/Radio \\
\hline
\end{tabular}

Figura 3. Modelo de máquina de estado finito AX.25 Fuente: Beech, Nielsen, \& Taylor (1997). 
físico, por lo que da la posibilidad de conmutar los enlaces en los tiempos en que sea requerido; existe una máquina de estado de multiplexado por cada canal físico que se vaya a utilizar. Por último, la máquina de estado físico se encarga de manipular tanto el transmisor como el receptor de radio (Beech, Nielsen, \& Taylor, 1997).

\section{Estructura de la trama AX.25}

Los enlaces de datos que utilizan paquetes de radio usan pequeños bloques de datos definidos como tramas. El protocolo AX.25 emplea tres tipos de trama. La figura 4a muestra la estructura de las tramas de supervisión ( $\mathrm{S}$ frame) y no numerada $(\mathrm{U}$ frame), y la figura $4 \mathrm{~b}$ muestra la estructura de la trama de información (I frame).

Cada campo dentro de la trama está formado por un número entero de octetos, y la función que cumple se describe a continuación.

El campo de bandera tiene una longitud de un octeto, cada trama debe iniciar y terminar con una bandera con el fin de poder delimitar las tramas, similar a HDLS (Joongs, 1982); la bandera se conforma del octeto 01111110 o el valor 7E hexadecimal. El siguiente campo corresponde a las direcciones tanto de origen como destino. El campo de control identifica el tipo de trama que se esté transmitiendo y administra los atributos de la conexión de la capa 2. La sección de identificación de protocolo (PID) aparece únicamente en las tramas de información y se encarga de identificar el tipo de protocolo de capa 3, si se encuentra presente y si está en uso, mientras que el campo de información transmite los datos de usuario de extremo a extremo del enlace.

La trama del protocolo AX.25 contiene un espacio dedicado a la secuencia de chequeo de trama (FCS), el cual es un dato de 16 bits que se calcula tanto en emisor como receptor; la función de este campo es asegurar que la información no ha sufrido modificaciones causadas por el medio de transmisión; el dato para realizar la secuencia de chequeo de trama se calcula de acuerdo con las recomendaciones establecidas en el documento de referencia HDLC, ISO 3309.

En el proceso de transmisión, en el campo de la trama correspondiente a la secuencia de chequeo, se envía primero el bit más significativo; en los demás campos se envía primero el bit menos significativo. Las solicitudes de servicios de enlace que requiere la capa 3 se realizan utilizando las interacciones de comando-respuesta que se denominan como primitivas y se encuentran los siguientes tipos: primitiva REQUEST, usada por la capa superior para solicitar servicio de enlace; primitiva INDICATION, que notifica a la capa superior una actividad específica relacionada con el servicio solicitado; primitiva RESPONSE, utilizada por una capa para acusar el recibo de la primitiva de INDICATION y la primitiva CONFIRM, que confirma que la actividad ha sido completada. En la figura 5 se muestra un ejemplo del proceso utilizando primitivas.

En términos de eficiencia, la configuración del protocolo AX.25 depende de muchos parámetros,

\begin{tabular}{|c|c|c|c|c|c|}
\hline Flag & Address & Control & Info & FCS & Flag \\
\hline 01111110 & $112 / 224$ Bits & $8 / 16$ Bits & N*8 Bits & 16 Bits & 01111110 \\
\hline
\end{tabular}

(a)

\begin{tabular}{|c|c|c|c|c|c|c|}
\hline Flag & Address & Control & PID & Info & FCS & Flag \\
\hline 01111110 & $112 / 224$ Bits & $8 / 16$ Bits & 8 Bits & N*8 Bits & 16 Bits & 01111110 \\
\hline
\end{tabular}

(b)

Figura 4. (a) Estructura de las tramas $S$ (supervisión) y U (no numerada). (b) Estructura de la trama I (información).

Fuente: Beech, Nielsen, \& Taylor, 1997. 
los cuales se ajustan de acuerdo con el software del controlador de nodo terminal (TNC); los siguientes son los parámetros más importantes:

- Tamaño de ventana: en la versión 2.2 del protocolo, el número máximo de tramas enviadas consecutivamente antes de esperar el acuse de recibido no puede superar una longitud de 127 bits; este parámetro se denota como $k$.

- Capacidad máxima en el campo de datos: una trama de información o una trama no numerada no debe superar los 256 bits de longitud; este parámetro se denota como $N_{1}$.

- Tiempo transcurrido entre el fin de la última trama l y el acuse de recibido: este parámetro puede o no ocurrir durante la transmisión. Dependiendo del software del TNC, puede ser configurado manual o automáticamente; se denota como $T_{2}$.

- Tiempo de estabilización de los parámetros del transmisor después de iniciar la transmisión y detección de la portadora en el receptor: depende de la capacidad del equipo y puede variar entre 10 y varios cientos de milisegundos; se denota como $T_{103}$.

- Duración de chequeo de portadora basado en prevención de colisiones: típicamente este tiempo es de 50 a 300 milisegundos; se denota como $T_{102}$.

- Persistencia de chequeo de portadora basado en prevención de colisiones: no se encuentra dentro de la descripción del protocolo, pero es implementado en la mayoría de software (Zielinski, 2009); típicamente es igual a 64, que significa una probabilidad de transmisión del 25\%; se denota como $p$.

El proceso de transmisión es iniciado por parte del TNC verificando la disponibilidad del canal para establecer el enlace, esta tarea se relaciona con el parámetro $p$, a partir del cual es posible calcular retardo promedio de acceso al canal utilizando la ecuación (1) (Zielinski, 2009).

$$
T_{C S}=\frac{256 T_{102}}{2(p+1)}
$$

Con el equipo transmisor encendido, el TNC espera el tiempo de estabilización, $T_{103^{\prime}}$ con el fin de asegurar que el proceso de transmisión se llevará a cabo de manera estable. Si el valor $k$ es mayor a 1 y se cuenta con suficientes tramas para la transmisión, varias tramas de información se pueden enviar de manera simultánea. En el momento de detener la transmisión, el receptor espera para cerciorarse de que no hay más tramas de información, paso seguido espera $T_{103}$ y envía la trama RR (acuse de recibido) (Beech, Nielsen, \& Taylor, 1997). En la figura 6 se muestra el ejemplo del proceso de transmisión con respecto a una línea de tiempo y asumiendo un tamaño $k$ de 4.

En el proceso de transmisión, el protocolo AX.25 utiliza tramas específicas para apoyar las funciones de control del enlace de comunicaciones, estas

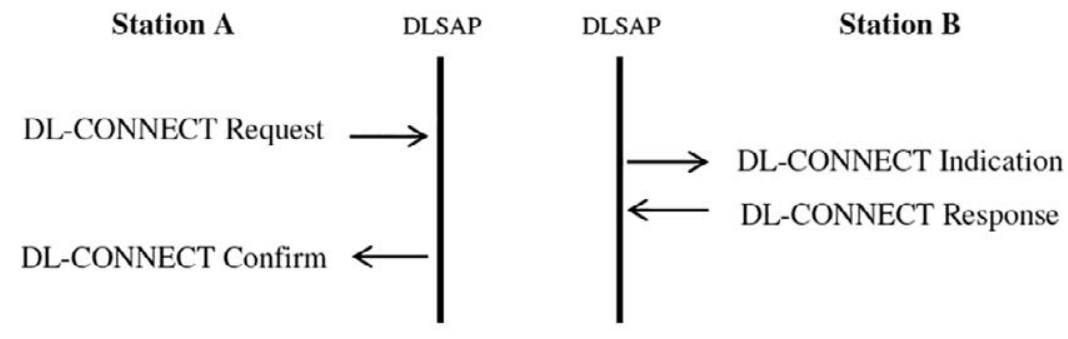

Figura 5. Uso de primitivas

Fuente: Beech, Nielsen, \& Taylor (1997). 
tramas según el estándar son (Beech, Nielsen, \& Taylor, 1997).

- RR: Receptor está listo

- RNR: Receptor no está listo

- REJ: Error en la transmisión

- FRMR: Informe de recibo de trama no esperada

\section{MATERIALES Y MÉTODOS}

El procedimiento desarrollado se llevó a cabo, inicialmente, recopilando la información y parámetros que definen el protocolo AX.25 y la forma de realizar enlaces de comunicación; posteriormente, con base en el trabajo desarrollado por Zielinski (2009), se realizó un análisis matemático de la velocidad de transmisión que se maneja en el protocolo AX.25.

Para el análisis matemático fue necesario implementar los modelos presentados por Joongs (1982), definiendo parámetros en las ecuaciones de acuerdo con el sistema de comunicación implementado en la estación terrena satelital UPTC. Utilizando el software Matlab se pudo obtener una representación gráfica del comportamiento en velocidad del sistema de comunicación implementado en la estación.

Una vez efectuado el análisis de simulación, se hizo necesario realizar pruebas y experimentos para comparar con la información obtenida en la simulación. El proceso de experimentación y las pruebas de campo se realizaron con la ayuda de los equipos con los que cuenta el Laboratorio de Electrónica y la estación terrena satelital de la UPTC, sede Tunja; en esta prueba se utilizó el vatímetro y el osciloscopio. El esquema de conexión que se utilizó para realizar las pruebas se muestra en la figura 7.

\section{PRESENTACIÓN DE RESULTADOS}

En esta sección se presentan los resultados de las pruebas realizadas, en primera instancia, utilizando las ecuaciones para obtener una estimación

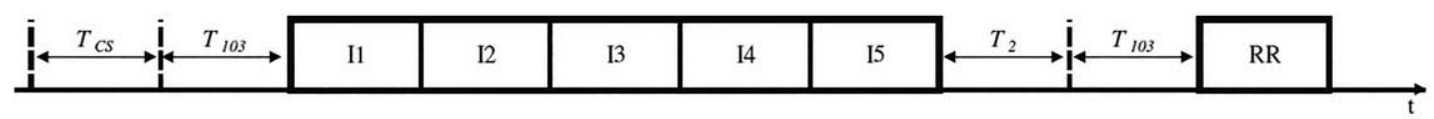

Figura 6. Secuencia de transmisión de 5 tramas de información

Fuente: Joongs (1982).

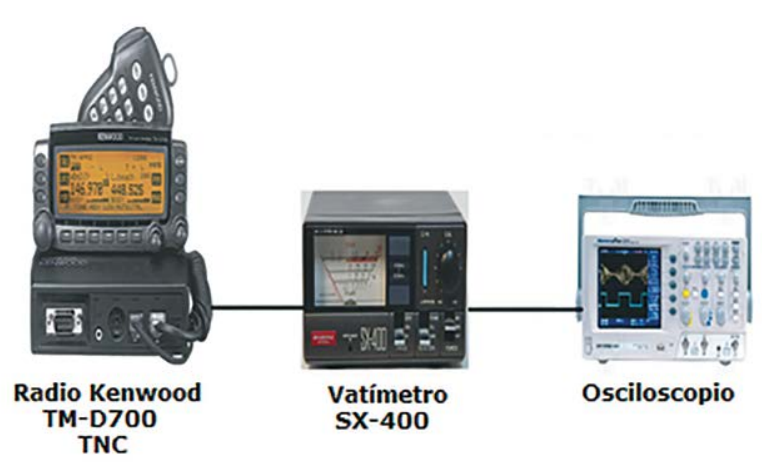

Figura 7. Conexión de equipos.

Fuente: elaboración propia. 
teórica de la velocidad de transmisión y posteriormente de forma práctica con el TNC del radio Kenwood TM-D700. Debido a las características del transceptor utilizado en la estación terrena satelital UPTC, no es posible realizar comunicaciones full dúplex utilizando protocolo AX.25.

El análisis teórico se basó en las ecuaciones planteadas por Zielinski (2009) para estimar la velocidad de transmisión del protocolo AX.25 configurado para comunicaciones de tipo half-duplex. Para realizar el análisis es necesario tener en cuenta los siguientes aspectos:

- El proceso de transmisión se realiza entre dos terminales.

- No se presentan colisiones o errores durante la transmisión.

- El tiempo de procesamiento de trama es despreciable.

- Los datos de entrada tienen longitud $L$.

- El enlace de radio opera a $R_{w l}$ bps.

- La trama para el acuse de recibido $R R$ tiene una longitud de 20 bytes.

- La trama de información está compuesta por 20 $+\mathrm{N} 1$ bytes.

Se consideraron los siguientes parámetros para el protocolo AX.25:

- $\quad N_{1}$ : capacidad máxima para el campo correspondiente a los datos

- $k$ : número máximo de tramas a enviar (tamaño de ventana)

- $T_{103}$ : tiempo de inicio para el transmisor

- $T_{2}$ : retardo de respuesta
El proceso de comunicación half-duplex en el protocolo AX.25 consiste en un conjunto de ciclos, donde cada ciclo se conforma de hasta $k$ tramas de información y un acuse de recibido $R R$. La cantidad de ciclos que se presenten dependerá de los parámetros de transmisión actuales del protocolo.

Inicialmente se tendrá en cuenta el tiempo de transmisión que emplea una trama considerando los retardos de bit que pueden generar incrementos en el tiempo de transmisión en un factor de 1/62, según Joongs (1982); a partir de esto, el tiempo de una trama de información se puede obtener a partir del modelo planteado por Zielinski (2009), ecuación (2).

$$
T_{I}=T_{103}+\frac{63}{62} * \frac{160+8 * N_{1}}{R_{w l}}
$$

Así mismo, el tiempo de transmisión para la trama RR se podrá estimar a partir de la ecuación (3).

$$
T_{R R}=T_{2}+T_{103}+\frac{63}{62} * \frac{160}{R_{w l}}
$$

Las ecuaciones (2) y (3) expresan el tiempo que está ocupado el canal de comunicación durante la transmisión de tramas de información, acorde con las suposiciones hechas, además incluyen el tiempo de puesta en marcha del transmisor.

Si se transmiten varias tramas de información, es decir, el dato de entrada tiene un tamaño de $N_{1} \times k$, la transmisión se llevará a cabo en varios ciclos, donde cada ciclo de transmisión enviará $k$ tramas de información y un solo acuse de recibido $R R$. Teniendo en cuenta las reglas de operación del protocolo (Zielinski, 2009), el tiempo total de transmisión se puede definir a partir de la ecuación (4).

$$
T_{P}=T_{2}+2 * T_{103}+(1+k) *\left(\frac{63}{62} * \frac{160}{R_{w l}}\right)+k *\left(\frac{63}{62} * \frac{8 * N_{1}}{R_{w l}}\right)
$$


En la ecuación (4), el primer componente hace referencia a la información propia del protocolo, mientras que el tiempo necesario para la transmisión de los datos como tal se relaciona con los demás términos en la ecuación.

A partir de las ecuaciones anteriores y de los parámetros que definen las reglas de operación del protocolo AX.25 se puede obtener una estimación de la velocidad efectiva de transmisión a la hora de implementar un enlace de comunicación utilizando el sistema de la estación terrena satelital UPTC. Los resultados se muestran en las figuras 8 y 9 .

En la figura 8 se observa que el protocolo AX.25 es más eficiente en cuanto a velocidad a medida que el número de tramas enviadas aumenta.

Caso similar se presenta al realizar el análisis configurando una velocidad de transmisión estimada de 9600 bps. El comportamiento de la velocidad efectiva en función de las tramas enviadas se muestra en la figura 9.

Utilizando el esquema de conexión de la figura 7 , se realizaron mediciones de las señales que entrega el transceptor Kenwood TM-D700; las mediciones se apoyaron en el osciloscopio y en el vatímetro SX-400. En la Figura 10 se muestran los

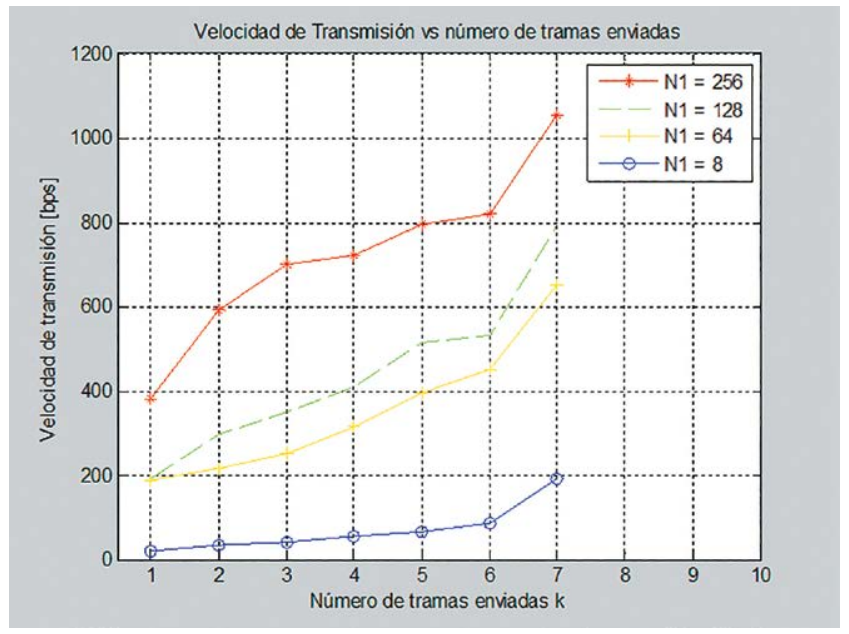

Figura 8. Velocidad de transmisión vs. Número de tramas enviadas a 1200 bps.

Fuente: elaboración propia. resultados obtenidos en esta prueba, a partir de la cual se obtuvo un tiempo de bit de 910 microsegundos para la configuración de 1200 bps, es decir, en esta configuración realmente se obtuvo una velocidad real de 1098,9 bps. En la configuración de 9600 bps se observó un tiempo de bit de 108.5 microsegundos, lo que significa una velocidad de transmisión real de 9216,59 bps.

El transceptor Kenwood TM-D700, con el cual se realizaron las pruebas, es el dispositivo que se encuentra instalado en el sistema de comunicaciones de la estación terrena satelital y cumple con la función de TNC (controlador de nodo terminal). Las características de este dispositivo se muestran en la tabla 1, mientras que la figura 11 muestra el panel del transceptor.

\section{DISCUSIÓN}

El desarrollo de este trabajo permitió obtener una mejor comprensión del protocolo de comunicación que se encuentra implementado en el sistema de comunicación de la estación terrena satelital UPTC. Este estudio se sustenta en el análisis del modelo matemático que describe los tiempos y la velocidad de

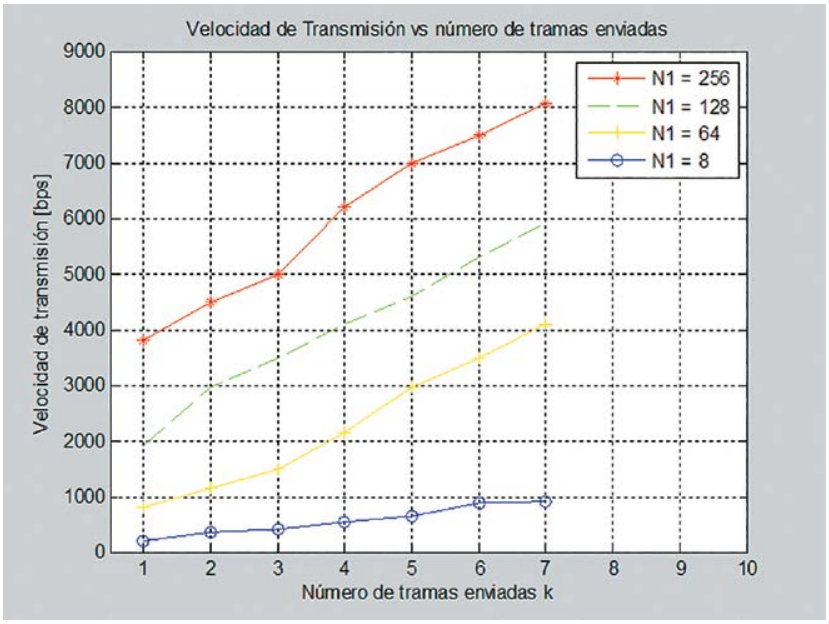

Figura 9. Velocidad de transmisión vs. número de tramas enviadas a 9600 bps.

Fuente: elaboración propia. 


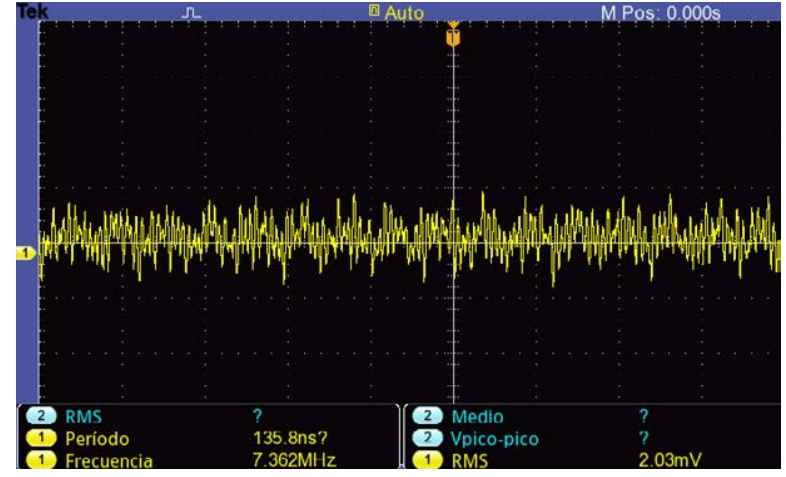

Figura 10. Mediciones realizadas con el osciloscopio.

Fuente: elaboración propia.

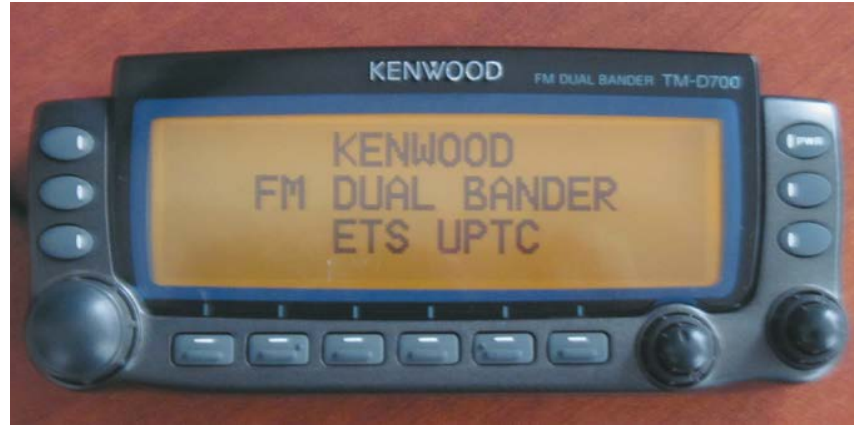

Figura 11. Fotografía de interfaz TNC.

Fuente: elaboración propia.

Tabla 1. Parametros del transceptor Kenwood TM-D700

\begin{tabular}{clll}
\hline Ítem & \multicolumn{1}{c}{ Característica } & \multicolumn{1}{c}{ Referencia } & \multicolumn{1}{c}{ Valor } \\
\hline 1 & Unidad TNC & X52-3310-00 & $45,05 \mathrm{MHz}$. (frecuencia de reloj). \\
\hline 2 & Ancho de banda & No aplica & $\pm 7,5 \mathrm{KHz}$ \\
\hline 3 & Perdidas por inserción & No aplica & $6 \mathrm{~dB}$ \\
\hline 4 & Impedancia de salida & No aplica & $800 \Omega \pm 10 \%, 2 \mathrm{pF} \pm 10 \%$ \\
\hline 5 & Banda de operación & VHF/UHF & $144-146 \mathrm{MHz} / 430-440 \mathrm{MHz}$ \\
\hline
\end{tabular}

Fuente: elaboración propia

transmisión en un enlace de comunicación utilizando el protocolo AX.25. Además, a partir del análisis teórico y las mediciones hechas, se pudieron comparar los resultados obtenidos y verificar una aproximación al modelo propuesto por Zielinski (2009), respecto a los experimentos y pruebas realizadas con los equipos de la estación terrena satelital UPTC.

El resultado obtenido es un aporte al área de las comunicaciones satelitales en Colombia, específicamente en la región de Boyacá; estos resultados se relacionan con los trabajos de Ekici, Akyildiz, \& Bender (2001), De Cola \& Marchese (2005), Diersing \& Ward (1989), Parry, (1997) y Havlicek, McKeeman, \& Remaklus (1995), los cuales presentan análisis de protocolos y sistemas enfocados en comunicaciones satelitales utilizando satélites LEO.

El análisis presentado en este artículo permite hacer predicciones sobre posibles aplicaciones que puedan ser implementadas utilizando el sistema de comunicación de la estación terrena satelital UPTC, pues es posible adaptar y codificar los datos de tal forma que se optimice el uso del protocolo AX.25. Ejemplos como los que se presentan en Henderson \& Katz (1999), Lasorso, Garrett, \& Patterson (2009), Martin, Venkatesan, \& Das (2003), Negoda, Bunin, Bushuev, \& Dranovsky (1997), Ronan, Walsh, \& Long (2010) y Sklyarevich (1992) evalúan el desempeño de protocolos de comunicación como el AX.25 en aplicaciones puntuales en el contexto de comunicaciones espaciales.

Este artículo sugiere un avance en el estudio de las comunicaciones satelitales, ya que en la región de Tunja no se ha realizado un estudio similar; también permite un mejor conocimiento de las variables que influyen en la velocidad de transmisión utilizando el sistema AX.25 de la estación terrena satelital UPTC. 


\section{CONCLUSIONES}

Se concluye que el hardware utilizado para la transmisión influye en la velocidad de transmisión, debido a que si se presentan cambios en el hardware se producen alteraciones en los tiempos de inicio en el transmisor, los cuales inmediatamente cambian los tiempos totales de transmisión; esto se debe a que los fabricantes de hardware tienen diferentes tipos de componentes y microprocesadores para los TNC.

Los resultados obtenidos a partir de las ecuaciones teóricas se aproximan bastante a los resultados reales cuando se efectúan transmisiones. Además del hardware utilizado, la velocidad de transmisión depende de la cantidad de tramas de información que se quieran enviar al receptor, puesto que el protocolo AX.25 es muy eficiente a nivel de hardware.

\section{AGRADECIMIENTOS}

A la Universidad Pedagógica y Tecnológica de Colombia y al Grupo de Investigación en Informática, Electrónica y Comunicaciones INFELCOM por su colaboración y el préstamo de algunos equipos e instalaciones requeridos en la realización del proyecto.

\section{REFERENCIAS}

Beech, W. A., Nielsen, D. E., \& Taylor, J. (1997). Link Access Protocol Amateur Packet Radio. Tucson: Tucson Amateur Packet Radio Corporation.

De Cola, T., \& Marchese, M. (2005). Performance Analysis of Data Transfer Protocols over Space Communications. IEEE Transactions on Aerospace and Electronic Systems, 1200-1223.

Diersing, R. J., \& Ward, J. W. (1989). Packet Radio in the Amateur Satellite Service. IEEE Journal on Selected Areas in Communications, 226-234.

Ekici, E., Akyildiz, I., \& Bender, M. (2001). A Distributed Routing Algorithm for Datagram Traffic in LEO Satellite Networks. IEEE/ACM Transactions on Networking 9, 137-147.
Espindola, J., Nonsoque, C., \& Molano, D. (2012). Montaje e implementación de una estación terrena satelital para el seguimiento de satélites de orbita baja. Latin American and Caribbean Conference for Engineering and Technology LACCEI. Panamá.

Havlicek, J. P., McKeeman, J. C., \& Remaklus, P. W. (1995). Networks of low-Earth Orbit Store-andforward Satellites. IEEE Transactions on Aerospace and Electronic Systems, 543-554.

Henderson, T., \& Katz, R. (1999). Transport Protocols for Internet-Compatible Satellite Networks. IEEE Journal on Selected Areas in Communications, 326-343.

Joongs, S. M. (1982). On the Impact of HDLC Zero Insertion and Deletion on Link Utilization and Reliability. IEEE Transactions on Communications, 375-381.

Lasorso, D., Garrett, P., \& Patterson, C. (2009). Protocol Considerations to Improve the Reliability of Data Collection in a Radio Telemetry System. World Environmental and Water Resources Congress 2009, (págs. 2220-2235). Great Rivers.

Martin, K., Venkatesan, V., \& Das., U. (2003). Data Link Control in the LEO Satellite Store and Forward Network. IEEE Region 10 Annual International Conference, (pp. 1234-1238).

Negoda, A., Bunin, S., Bushuev, E., \& Dranovsky, V. (1997). LEOPACK The Integrated Services Communications System Based on LEO Satellites. Acta Astronautica, 661-669.

Parry, R. R. (1997). AX.25. IEEE Potentials 16, 14-16.

Ronan, J., Walsh, K., \& Long, D. (2010). "Evaluation of a DTN Convergence Layer for the AX.25 Network Protocol. 2nd International Workshop on Mobile Opportunistic Networking, (pp. 72-77).

Sklyarevich, A. (1992). Performance of an X.25 Channel with Overlapping Error Bursts in Transmitting a Large Data Array. Automatic Control and Computer Sciences, 21-31.

Zielinski, B. M. (2009). Effective Transmission Speed in AX.25 Protocol. EUROCON 2009, EUROCON '09. IEEE (págs. 1763-1768). IEEE.

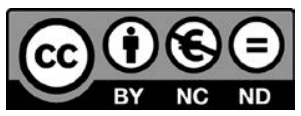

Tecnura • p-ISSN: 0123-921X • e-ISSN: 2248-7638 • Vol. 19 No. $46 \bullet$ Octubre - Diciembre $2015 \bullet$ pp. 93-102 [ 102 ] 